\title{
Teoremas de puntos fijos para correspondencias con dominios no compactos y no convexos
}

\author{
Theorems of Fixed Points for Correspondences with non-Compact and non- \\ Convexed Domains
}

\author{
${ }^{1}$ Valerio Bonifacio Chávez Anticona.
}

\begin{abstract}
RESUMEN
Este documento proporciona algunos teoremas de puntos fijos para correspondencias semicontinuas inferiores y superiores, y correspondencias inferiores abiertas definidas en conjuntos no compactos y no convexos. Se observará que las condiciones de nuestros teoremas no solo son suficientes sino también necesarios. Además, nuestros teoremas generalizan algunos teoremas de punto fijo conocidos, como el teorema de punto fijo de Kakutani y el teorema de punto fijo de Brouwer-Schauder al relajar las condiciones de compacidad y convexidad.
\end{abstract}

Palabras clave: compacto, convexo, correspondencia, punto fijo, semicontinua.

\begin{abstract}
This document provides some fixed point theorems for lower and upper semicontinuous correspondences, and open lower correspondences defined in non-compact and non-convex sets. It will be observed that the conditions of our theorems are not only sufficient but also necessary. In addition, our theorems generalize some known fixed point theorems, such as the Kakutani fixed point theorem and the Brouwer-Schauder fixed point theorem by relaxing the conditions of compactness and convexity.
\end{abstract}

Keywords: compact, convex, correspondence, fixed point, semi-continuous.

\section{INTRODUCCIÓN}

Los teoremas de punto fijo son las herramientas matemáticas básicas utilizadas para demostrar la existencia de conceptos de solución en teoría de juegos y economía (Border, 1989; Debreu, 1952). En particular, son herramientas matemáticas clave para demostrar la existencia del equilibrio. Sin embargo, la mayoría de los teoremas de punto fijo en la literatura (Border, 1989; Joshi \& Bose, 1985) se demuestran sobre conjuntos convexos, compactos y solo se dan condiciones suficientes para la existencia de puntos fijos de correspondencias. Además, los teoremas de punto fijo obtenidos por (Halpern, 1970; Llerena, 2016; Tian, 1991), suponen que las correspondencias internas (débilmente) son semicontinuas superiores o demicontinuas superiores. El propósito de este documento es presentar algunas condiciones necesarias y suficientes para la existencia de puntos fijos de correspondencias semicontinuas inferiores y correspondencias semicontinuas superiores y correspondencias con secciones inferiores abiertas definidas en conjuntos no compactos y no convexos en un espacio vectorial topológico de Fréchet y espacios localmente convexos en un espacio vectorial topológico de Hausdorff. Se observará que estos resultados generalizan el teorema del punto fijo de Kakutani y el teorema del punto fijo de BrouwerSchauder al relajar las condiciones de compacidad y convexidad. Aunque este documento solo considera la existencia de puntos fijos de las correspondencias semicontinuas inferiores y superiores y de las secciones inferiores abiertas, creemos que algunas de las técnicas desarrolladas en el documento pueden aplicarse para extender otros teoremas de puntos fijos.

'Universidad Nacional Jorge Basadre Grohmann. Tacna - Perú. E-mail: vchaveza@unjbg.edu.pe 


\section{NOTACIÓN Y DEFINICIONES}

Sea $X$ un conjunto. Designaremos por $P(X)$ la familia de todos los subconjuntos de $X$ y, en particular, el conjunto vacío $\emptyset$; y el conjunto total $X$. Una topología en $X$ es una familia de subconjuntos de $X, \tau \subset P(X)$, que cumple

(T1) $\varnothing, X \in \tau$

(T2) $\quad \mathrm{Si} U_{1}, U_{2}, \cdots, U_{k} \in \tau$, también $U_{1} \cap$ $U_{2} \cap \cdots \cap U_{k} \in \tau$

(T3) $\quad$ Si $U_{j} \in \tau$ para todo $j \in \mathrm{J}$ con J familia arbitraria de índices, entonces $\bigcup_{j \in \mathrm{J}} U_{j} \in \tau$.

(Llerena, 2016)

Un espacio topológico $(X ; \tau)$ es un conjunto $X$ junto con una topología $\tau$ en $X$. Los elementos de $\tau$ se llaman abiertos; los elementos de $X$ se llaman puntos (Llerena, 2016).

Sean $X$ y $Y$ dos espacios topológicos, y sea $2^{Y}$ todos los subcojuntos de $Y$. Una correspondencia $G: X \rightarrow 2^{Y}$ se dice que es semicontinua superior (en resumen, s.c.s.) si el conjunto $\{x \in$ $X: G(x) \subset V\}$ es abierto en $X$ para cada subconjunto abierto $V$ de $Y$ (Narváez \& Restrepo, 2013; Tian, 1991).

Una correspondencia $G: X \rightarrow 2^{Y}$ se dice que es semicontinua inferior (en resumen, s.c.i.) si el conjunto $\{x \in X: G(x) \cap V \neq \varnothing\}$ es abierto en $X$ para cada subconjunto abierto $V$ de $Y$ (Narváez $\&$ Restrepo, 2013; Tian, 1991).

Una correspondencia $G: X \rightarrow 2^{Y}$ se dice que tiene secciones superiores abiertas si, para cada $x \in X, G(x)$ es abierto en $Y$ (Tian, 1991). Una correspondencia $G: X \rightarrow 2^{Y}$ se dice que tiene secciones inferiores abiertas si el conjunto $G^{-1}(y)=\{x \in X: y \in G(x)\}$ es abierto en $X$ para cada $y \in Y$ (Choban, 2014; Tian, 1991).

Un espacio vectorial topológico es un conjunto con dos estructuras compatibles. Por un lado, tiene la estructura algebraica de un espacio vectorial y, por otro, tiene una topología, por lo que las nociones de convergencia y continuidad son significativas. Estas dos estructuras deben ser compatibles en el sentido de que las operaciones algebraicas son continuas. (Lambers, 2011)

Sea $X$ un espacio vectorial sobre el campo real o complejo $\Phi$. Se dice que una topología $\tau$ en $X$ es compatible con la estructura algebraica de $X$ si las operaciones algebraicas en $X$ son continuas, es decir, $x+y$ es una función continua del par de del producto $\tau x \tau$ y $\mathbb{C} x X$ con la topología del producto $\mathcal{C} x \tau$, donde $\mathcal{C}$ es la topología habitual en el plano complejo $\mathbb{C}$. Recordamos el significado de una "topología del producto".

Considere dos espacios topológicos $X$ y $Y$. Para decir cuál es la topología del producto en $X x Y$, es suficiente exhibir una base de vecindades de cada punto $(x ; y)$ de $X x Y$. Tal base es proporcionada por los rectángulos

variables $x, y$ y $\lambda x$, es una función continua del par de variables $\lambda, x$. Un espacio vectorial topológico sobre $\Phi$ es un espacio vectorial sobre $\Phi$ con una topología compatible. (Lambers, 2011)

Un conjunto $C \subseteq \mathbb{R}^{n}$ es convexo si el segmento de línea entre dos puntos en $C$ también se encuentra en $C$. Dados dos vectores $x, y \in \mathbb{R}^{n}$, el segmento de línea entre $x$ e $y$ está definido por

$$
[x ; y]=\{x+\lambda(y-x) / 0 \leq \lambda \leq 1\},
$$

que puede reescribirse como

$$
[x ; y]=\{\lambda y+(1-\lambda) x / 0 \leq \lambda \leq 1\} .
$$

(Lambers, 2011)

Un espacio métrico es un par $(X ; d)$ donde $X$ es un conjunto y $d$ es una función llamada función de distancia o métrica definida de $X x X$ en $\mathbb{R}$, la cual satisface las siguientes condiciones para $x$, $y, z \in X$ :

$$
\begin{aligned}
d(x ; y) \geq & 0 \\
d(x ; y)= & 0 \Leftrightarrow x=y \\
d(x ; y)= & d(y ; x) \\
& d(x ; z) \leq d(x ; y)+d(y ; z)
\end{aligned}
$$

(Márquez, 2009)

El conjunto de los números complejos $\mathbb{C}$ con la función de distancia $d$ definida como $d(x ; y)=$ $|x-y|$, la cual constituye ser la topología habitual del plano complejo $\mathbb{C}$ (Márquez, 2009).

Sea $X$ un espacio vectorial sobre el campo de los números complejos $\mathbb{C}$ (en resumen, un espacio vectorial). Definamos

$$
\begin{gathered}
A_{v}: X x X \rightarrow X, A_{v}(x ; y)=x+y, \\
M_{s}: \mathbb{C} x X \rightarrow X, M_{s}(\lambda ; y)=\lambda . y,
\end{gathered}
$$


es la suma vectorial y la multiplicación escalar en $X$. Se dice que una topología $\tau$ en $X$ es compatible con la estructura lineal de $X$ si $A_{v}$ y $M_{S}$ son continuas cuando proveemos a $X$ con la topología $\tau, X x X$ con la topología

$$
U x V=\left\{\left(x^{\prime} ; y^{\prime}\right) \in X x Y: x^{\prime} \in U, y^{\prime} \in V\right\},
$$

donde $U$ (respectivamente $V$ ) es una vecindad de $x$ (respectivamente $y$ ) en $X$ (respectivamente $Y$ ). Que estos rectángulos formen una base de filtro es trivial; obviamente no forman un filtro (excepto en casos triviales), ya que un conjunto que contiene un rectángulo no tiene que ser un rectángulo. Queda por comprobar que los filtros así definidos, para cada par $(x ; y)$, de hecho, se puedan tomar como filtros de vecindades de $(x ; y)$ en una topología en $X x Y$. La topología $\mathcal{C}$ asigna a cada punto $\lambda$ del plano complejo una base notable de vecindades, los discos, abiertos o cerrados, con centro en este punto (y con radio positivo $r$ ). Cuando se le proporciona una topología compatible con su estructura lineal, $X$ se convierte en un espacio vectorial topológico, que abreviaremos en EVT. (Treves, 2016)

Se dice que un espacio topológico $X$ es de Hausdorff si, dados dos puntos distintos $x$ e $y$ de $X$, existe una vecindad $U$ de $x$ y una vecindad $V$ de $y$ que no se intersecan, es decir, que $U \cap V=$ $\emptyset$. Una propiedad muy importante de los espacios topológicos de Hausdorff es la llamada unicidad del límite: Un filtro en un espacio topológico de Hausdorff $X$ converge como máximo en un punto. (Treves, 2016)

Sea $D$ un subconjunto convexo no vacío del espacio vectorial topológico $X$ de Hausdorff localmente convexo y sea $I_{D}(x)=\{y \in E: \lambda x+$ $(1-\lambda) y \in D \quad y \quad \lambda \in[0 ; 1)\}$. Una correspondencia $G: D \rightarrow 2^{E}$ se dice que es interior en $D$ si $G(x) \cap I_{D}(x) \neq \varnothing$ para todo $x \in$ $D$, o equivalentemente, para cada $x \in D$, existe un $y \in G(x)$ y $\lambda \in[0 ; 1)$ tal que $\lambda x+(1-$ $\lambda) y \in D$. Una correspondencia $G: D \rightarrow 2^{E}$ se dice que es débilmente interior en $D$ si $G(x) \cap$ $\operatorname{clI}_{D}(x) \neq \varnothing$. (Tian, 1991)

Una función no-negativa $\|$.$\| en un espacio$ vectorial (es decir, lineal) $X$ se llama norma en $X$ si $\|x\| \geq 0$ para cada $x \in X$,

$T: M \rightarrow M$ es compacta, entonces $T$ tiene un punto fijo (Smith, 2014).

Teorema (Teorema de punto fijo de Brouwer). Sea $D \subseteq \mathbb{R}^{2}$. Dada la función $f: D \rightarrow D$ continua, entonces existe algún $c \in D$ tal que $f(c)=c$; es decir, $c$; es un punto fijo. Donde $D$ es un disco cerrado unitario (Buxton, 2016).

Teorema [Teorema de punto fijo de Kakutani]. Si $X$ es un simplex y $F: X \rightarrow X$ es una correspondencia semicontinua superior con valores convexos compactos no vacíos (llamada correspondencia Kakutani), entonces existe un $x^{*} \in X$ tal que $x^{*} \in F\left(x^{*}\right)$ (Lassonde, 1990).

Un espacio topológico es llamado paracompacto si cada cubrimiento abierto admite un refinamiento abierto localmente finito. Un refinamiento de un cubrimiento $U$ siempre significa un cubrimiento que refina $\mathcal{U}$ (Ergun, 1980).

$\|x\|=0$ si y solo si $x=0$,

$\|\lambda x\|=|\lambda|\|x\|$ para cada $x \in X$ y para cada escalar $\lambda$,

$\|x+y\| \leq\|x\|+\|y\|$ para cada $x ; y \in X$ (la "desigualdad triangular").

Un espacio vectorial $X$ con una norma $\|$.$\| es$ denotado por $(X ;\|\|$.$) , es llamado un espacio$ lineal normado (o simplemente un espacio normado). (Fabian, et al., 2011)

Se debe tener en cuenta que la función $\rho(x ; y):=$ $\|x-y\|$, donde $x ; y \in X$, es de hecho una métrica en $X$.

Un espacio de Banach es un espacio lineal normalizado $(X ;\|\|$.$) , que es completo en la$ métrica canónica definida por $\rho(x ; y)=\| x-$ $y \|$ para $x ; y \in X$, es decir, cada sucesión de Cauchy en $X$ para la métrica $\rho$ converge en algún punto de $X$ (Fabian, et al., 2011).

Sea $(X ; \tau)$ un espacio vectorial topológico sobre $\mathbb{K}$. $(X ; \tau)$ es llamado $F$ - espacio si, y solo si, $\tau$ es inducida por una métrica invariante de traslación completa en $X$. Por otro lado $(X ; \tau)$ es llamado espacio Fréchet si, y solo si, es un $F$ - espacio localmente convexo. (Philip, 2019) 
Teorema (Teorema de selección semilineal de Michael). Supongamos que $T$ es s.c.i. con valores convexos cerrados, y el dominio $M$ de $X$ es cerrado y acotado. Entonces $T$ tiene una selección semilineal continua (Aschenbrenner \& Thamrongthanyalak 2015).

Teorema (Teorema de punto fijo de Schauder's). Sea $X$ un espacio de Banach y sea $M \subseteq X$ no vacío, convexo y cerrado. Si

Sea $D^{n}=\left\{x \in \mathbb{R}^{n}:\|x\| \leq 1\right\}$. Un subconjunto $E \subset \mathbb{R}^{n}$ se llama retracción de $D^{n}$ si existe una función continua $r: D^{n} \rightarrow E \quad$ (llamada retracción) tal que $r(x)=x$ para cada $x \in E$.

Teorema [Brouwer]. Sea $f: D^{n} \rightarrow D^{n}$ una función continua. Entonces $f$ tiene un punto fijo $x^{*} \in D^{n}$ (Pata, 2014).

\section{LA EXISTENCIA DE PUNTOS FIJOS DE CORRESPONDENCIAS}

Antes de pasar a los teoremas principales, establecemos algunos lemas técnicos que fueron establecidos por (Michael, 1956; Yannelis \& Prabhakar, 1983).

Lema 1. Sean $X$ y $Y$ dos espacios topológicos y sean $\phi: X \rightarrow 2^{Y}$ y $\psi: X \rightarrow 2^{Y}$ correspondencias tal que

$\phi$ es s.c.i. y tiene secciones superiores abiertas,

$\psi$ es s.c.i.,

para cada $x \in X, \phi(x) \cap \psi(x) \neq \emptyset$.

Entonces la correspondencia $\theta: X \rightarrow 2^{Y}$ definida por $\theta(x)=\phi(x) \cap \psi(x)$ es s.c.i.

Lema 2. Sean $X$ y $Y$ dos espacios topológicos y sean $\phi: X \rightarrow 2^{Y}$ y $\psi: X \rightarrow 2^{Y}$ correspondencias que tienen secciones inferiores abiertas. Entonces la correspondencia $\theta: X \rightarrow 2^{Y}$ definida por $\theta(x)=\phi(x) \cap \psi(x)$ tiene secciones inferiores abiertas.

Nuestros resultados principales es el siguiente el cual proporciona las condiciones necesarias y suficientes para la existencia de puntos fijos de correspondencias.
Teorema 1. Sea $X$ un subconjunto no vacío en un espacio vectorial topológico de Fréchet $E$. Supongamos que $F: X \rightarrow 2^{E}$ es una correspondencia semicontinua inferior con valores convexos cerrados no vacíos. Entonces la condición necesaria y suficiente para la existencia de un punto fijo $x^{*} \in F\left(x^{*}\right)$ es que existe un subconjunto convexo compacto no vacío $C \subset X$ tal que

$$
F(x) \cap C \neq \varnothing \quad \forall x \in C .
$$

$f\left(x^{*}\right) \in K\left(x^{*}\right) \subset F\left(x^{*}\right)$ y así $F$ tiene un punto fijo $x^{*} \in F\left(x^{*}\right)$.

Observación 1. Aun cuando $F$ es s.c.i. y $C$ es un subconjunto compacto y convexo no vacío, la correspondencia $K: C \rightarrow 2^{C}$ definida por $K(x)=F(x) \cap C$ para cada $x \in C$ no es necesario ser s.c.i. en $C$ si no es cierto $K(x) \neq \varnothing$ para toda $x \in C$ (Tian, 1991).

El siguiente ejemplo simple ilustra esto.

Ejemplo 1. Sea $X=[0 ; 3]$ y $Y=[1 ; 5]$. Sea $F: X \rightarrow 2^{Y}$ definida por $F(x)=[1+x ; 2+x]$. Si consideramos $C=[0 ; 2]$, entonces $K$ no es s.c.i. en $C$ siendo $\{x \in C: F(x) \cap C \neq \emptyset\}=$ $[0 ; 1]$ es no abierto en $C$.

Observación 2. Tenga en cuenta que la condición (1) implica que la correspondencia $F$ es interna en $C$ puesto que $C \subset I_{C}(x)$ para toda $x \in C$.

El teorema 1 requiere que $X$ sea un subconjunto no vacío en un espacio vectorial topológico de Fréchet. Podemos extender el teorema anterior a un subconjunto paracompacto no vacío en un espacio topológico localmente convexo de Hausdorff si $F$ se fortalece para tener secciones inferiores abiertas (Yannelis \& Prabhakar, 1983).

Demostración. Condición necesaria. Supongamos que $F$ tiene un punto fijo $x^{*} \in$ $F\left(x^{*}\right)$. Sea $C=\left\{x^{*}\right\}$. A continuación, tenemos que el conjunto unitario $C$ es claramente compacto y convexo tal que $F(x) \cap C \neq \varnothing$ para toda $x \in C$. 
Condición Suficiente. Supongamos que existe un conjunto convexo compacto no vacío $C$ tal que $F(x) \cap C \neq \varnothing$ para todo $x \in C$.

Definimos la correspondencia $K: C \rightarrow 2^{C}$ para cada $x \in C$, por,

$$
K(x)=F(x) \cap C .
$$

Puesto que la correspondencia $G: C \rightarrow 2^{C}$ definida por $G(x)=C$ para toda $x \in C$ es claramente s.c.i. y tiene secciones superiores abiertas en $C$ y $F$ es s.c.i., $K$ es s.c.i. por Lema 1. Note que también $K(x)$ en no vacío, compacto, $\mathrm{y}$ convexo para toda $x \in C$. Por lo tanto, según el teorema de selección de Michael (Aubin, 2007), existe una función continua $f: C \rightarrow C$ tal que $f(x) \in K(x)$ para todo $x \in C$. Ahora, al aplicar el teorema de punto fijo de Brouwer-Schauder (Aubin, 2007), existe un punto $x^{*} \in C$ tal que $x^{*}=$

Teorema 2. Sea $X$ un subconjunto de un paracompacto no vacío en un espacio vectorial topológico de Hausdorff localmente convexo $E$. Supóngase que $F: X \rightarrow 2^{E}$ tiene secciones inferiores abiertas tales que $F(x)$ es no vacío, cerrado y convexo par toda $x \in X$. Entonces la condición necesaria y suficiente para la existencia de un punto fijo $x^{*} \in F\left(x^{*}\right)$ es que exista un subconjunto convexo compacto no vacío $C \subset X$ tal que

$$
F(x) \cap C \neq \varnothing \quad \forall x \in C \text {. (3) }
$$

Demostración. La condición necesidad es la misma. La condición suficiente es similar a la anterior. Supongamos que existe un conjunto convexo compacto no vacío $C$ tal que $F(x) \cap$ $C \neq \varnothing$ para toda $x \in C$.

Definimos una correspondencia $K: C \rightarrow 2^{C}$ por, para cada $x \in C$,

$$
K(x)=F(x) \cap C .
$$

Luego, por el Lema 2, $K$ tiene secciones inferiores abiertas al notar que la correspondencia $G: C \rightarrow 2^{C}$ definida por $G(x)=C$ para toda $x \in C$ tiene secciones inferiores abiertas. $\mathrm{Y} K(x)$ es no vacío, compacto y convexo para todas $x \in C$. Por lo tanto, según
(Yannelis \& Prabhakar, 1983), existe una función continua $f: C \rightarrow C$ tal que $f(x) \in K(x)$ para toda $x \in C$. Por lo tanto, según el teorema de punto fijo de Brouwer-Schauder, existe un punto $x^{*} \in C$ tal que $x^{*}=f\left(x^{*}\right) \in K\left(x^{*}\right) \subset$ $F\left(x^{*}\right)$ y así $F$ tiene un punto fijo $x^{*} \in F\left(x^{*}\right)$.

Ahora damos un teorema similar para la correspondencia semicontinua superior.

Teorema 3. Sea $X$ un subconjunto no vacío en un espacio vectorial topológico de Hausdorff localmente convexo $E$. Supóngase que $F: X \rightarrow$ $2^{E}$ es una correspondencia semicontinua superior con valores convexos cerrados no vacíos. Entonces una condición necesaria y suficiente para la existencia de un punto fijo $x^{*} \in F\left(x^{*}\right)$ es que exista un subconjunto convexo compacto no vacío $C \subset X$ tal que

1979), existe $x^{*} \in C$ tal que $x^{*} \in K\left(x^{*}\right)$ y así $F$ tiene un punto fijo $x^{*} \in F\left(x^{*}\right)$.

El teorema 3 anterior puede extenderse al siguiente teorema que generaliza los teoremas de punto fijo dados en (Halpern, 1970; Halpern \& Bergman 1968) al relajar los conjuntos de compacidad y convexidad.

Teorema 4. Sea $X$ un subconjunto no vacío en un espacio vectorial topológico de Hausdorff convexo localmente $E$. Supongamos que $F: X \rightarrow$ $2^{E}$ es una correspondencia semicontinua superior con valores convexos cerrados no vacíos. Entonces la condición necesaria y suficiente para la existencia de un punto fijo $x^{*} \in F\left(x^{*}\right)$ es que existe un subconjunto convexo compacto no vacío $C \subset X$ tal que $F$ es débilmente hacia adentro en $C$.

Demostración. Solo tenemos que demostrar la suficiencia. Dado que $F$ es débilmente hacia dentro en el conjunto convexo compacto no vacío $C$ y $F: C \rightarrow 2^{E}$ es una correspondencia semicontinua superior con valores convexos cerrados, según el Teorema 2 en (Halpern, 1970), existen puntos fijos $x^{*} \in C$ tal que $x^{*} \in F\left(x^{*}\right)$.

Observación 3. Observe que en el caso de que $X$ sea un subconjunto convexo compacto no vacío y $F$ es una correspondencia de $X$ en $X$, las condiciones de suficiencia de los teoremas 1-3 se 
cumplen con $C=X$. Por lo tanto, los teoremas 2 y 3 generalizan el teorema 3.2. en (Yannelis \& Prabhakar, 1983) y el teorema de Kakutani fijado a conjuntos no compactos y no convexos.

$$
F(x) \cap C \neq \varnothing \quad \forall x \in C . \text { (5) }
$$

Demostración. Este teorema se puede demostrar aplicando el teorema 2 dado en (Halpern, 1970) notando $C \subset I_{C}(x)$ para toda $x \in C$. Aquí damos una demostración directa. Primero demostraremos que la correspondencia $K: C \rightarrow$ $2^{C}$ definida por $K(x)=F(x) \cap C$ para cada $x \in$ $C$ es s.c.s. De hecho, siendo $F$ una correspondencia semicontinua superior con valores convexos cerrados no vacíos, es cerrada (es decir, su gráfica es cerrada) por la Proposición 3.7 de (Aubin \& Ekeland, 1984). Por lo tanto, según el teorema 8 de (Aubin, 1979; Aubin \& Ekeland 1984), $K$ es una correspondencia semicontinua superior con valores compactos no vacíos. Por lo tanto, según el teorema de punto fijo de Kakutani (Aubin,

Observación 4. De las demostraciones de los teoremas 1 y 3 , podemos ver que aquí todavía existe un punto fijo de $F$ si la condición de que $F$ tiene valores convexos cerrados no vacíos en $X$ se debilita a la condición de que $F$ tenga valores convexos cerrados no vacíos en $C$.

Cuando una correspondencia se convierte en una función de un solo valor, tenemos el siguiente corolario al aplicar el Teorema 3:

Corolario 1. Sea $X$ un subconjunto no vacío en un espacio vectorial topológico de Hausdorff localmente convexo $E$. Supongamos que $f: X \rightarrow$ $E$ es una función continua. Entonces, la condición necesaria y suficiente para la existencia de un punto fijo $x^{*}=f\left(x^{*}\right)$ es que exista un subconjunto compacto convexo $C \subset X$ tal que

$$
f(x) \in C \quad \forall x \in C .
$$

Por lo tanto, el corolario anterior generaliza el teorema del punto fijo de Brouwer-Schauder al relajar las condiciones de compacidad $y$ convexidad.

Nuestros teoremas pueden demostrar la existencia de un punto fijo de una correspondencia que puede tener valores vacíos, no compactos o no convexos y el dominio puede ser no compacto y no convexo. El siguiente ejemplo simple ilustra esto.

Ejemplo 2. Sea $X=(-\infty ; a) \cup(b ;+\infty) \subset \mathbb{R}$, el cual es no compacto y no convexo. Aquí $0<$ $a<b$. Definimos una correspondencia semicontinua superior $F: X \rightarrow \mathbb{R}$ por, para cada $x \in C$,

$$
F(x)=(-\infty ; x-a] \cup[b ;+\infty),
$$

Lo cual es no compacto y no convexo. Por lo tanto, no podemos aplicar, digamos, el teorema del punto fijo de Kakutani para probar la existencia del punto fijo de $F$. Sin embargo, si tomamos $C=[b ; b+2]$, entonces $C$ es un intervalo compacto y convexo, $F$ es una correspondencia semicontinua superior con valores convexos compactos no vacíos en $C, \mathrm{y}$

Una correspondencia semicontinua superior e inferior la cual fue definida en dominios no convexos y no compactos también tienen puntos fijos.

\section{CONCLUSIONES}

Las correspondencias son generalizaciones de una función.

Los puntos fijos en las correspondencias tienen mucha aplicación tanto en el campo de las matemáticas, teoría de juegos y en la economía.

$$
F(x) \cap C \neq \varnothing \quad \forall x \in C .
$$

Así, por el teorema 3 y la observación $4, F$ tiene un punto fijo.

\section{RESULTADOS}

Una correspondencia semicontinua superior e inferior la cual fue definida en dominios convexos y compactos siempre tienen puntos fijos.

\section{REFERENCIAS BIBLIOGRÁFICAS}

Aschenbrenner, Matthias, and Athipat Thamrongthanyalak 2015 Michael's selection theorem in a semilinear context. Adv. Geom 15(3):293-313.

Aubin, Jean-Pierre $2007 \mathrm{Mathematical}$ methods of game and economic theory: Courier Corporation.

Aubin, JP 1979 Mathenutical Methods of Came and 
Economic Theory: North-Holland, Ameterdam., New York, Oxford.

Aubin, JP, and IEkeland $1984 \quad$ A p p 1 i e d Nonlinear Analysis John Wiley and Sons New York: NY

Border, Kim C 1989 Fixed point theorems with applications to economics and game theory: Cambridge university press.

Buxton, Colin 2016 Brouwer Fixed-Point Theorem.

Choban, Mitrofan M $2014 \quad$ Selections and fixed points theorems for mapping defined on convex spaces. ROMAI J 10:11-44.

Debreu, Gerard 1952 A social equilibrium existence theorem. Proceedings of the National Academy of Sciences 38(10):886893.

Ergun, N 1980 On nearly paracompact spaces. İstanbul University Science Faculty The Journal Of Mathematics, Physics and Astronomy 45:65-87.

Fabian, Marián, etal. 2011 B anach space theory: the basis for linear and nonlinear analysis: Springer Science \& Business Media.

Halpern, Benjamin $\quad 1970 \quad$ Fix ed p o in t theorems for set-valued maps in infinite dimensional spaces. Mathematische Annalen 189(2):87-98.

Halpern, Benjamin R, and George M Bergman 1968 A fixed-point theorem for inward and outward maps. Transactions of the American Mathematical Society 130(2):353358.

Joshi, Mohan C, and Ramendra K Bose 1985 Some topics in nonlinear functional analysis: John
Wiley \& Sons.

Lambers, Jim 2011 Summer Session.

Lassonde, Marc 1990 Fixed points for Kakutani factorizable multifunctions. Journal of Mathematical Analysis and Applications 152(1):46-60.

Llerena, Irene 2016 Topología.

$\begin{array}{lllll}\text { Márquez, Idelfonso Izquierdo } & 2 & 0 & 0 & 9\end{array}$ Introducción a la topologıa para análisis complejo.

Michael, Ernest 1956 Continuous selections. I. Annals of mathematics:361-382.

Narváez, Diana Ximena, and Guillermo Restrepo 2013 El Teorema de la Función Abierta para Funciones Multivaluadas Convexas. Revista de Ciencias 17(2):67-93.

Pata, Vittorino 2014 Fixed point theorems and applications. Politecnico di Milano.

Philip, Peter $2019 \quad$ Functional Analysis

Smith, Zachary 2014 Fixed Point Methods in Nonlinear Analysis. Online lecture notes, $\mathrm{m} \mathrm{a} \mathrm{t} \mathrm{h.} \mathrm{u} \mathrm{c} \mathrm{h} \mathrm{i} \mathrm{c} \mathrm{a} \mathrm{g} \mathrm{o.}$ edu/may/REU2014/REUPapers/Smith, Z. pdf.

Tian, Guoqiang 1991 Fixed points theorems for mappings with non-compact and non-convex domains. Journal of Mathematical Analysis and Applications 158(1):161-167.

Treves, François 2016 Topological Vector Spaces, Distributions and Kernels: Pure and Applied Mathematics. Volume 25: Elsevier.

Yannelis, Nicholas C, and ND Prabhakar 1983 Existence of maximal elements and equilibria in linear topological spaces. Journal of Mathematical Economics 12(3):233-245. 